\title{
RELACIONES ENTRE LA FILOSOFÍA DE HUME Y LA ÉTICA DE LA LEY NATURAL
}

\author{
FERNANDO ARANCIBIA C.* \\ Universidad Bernardo OHiggins \\ Pontificia Universidad Catolica de Chile
}

RESUMEN: La filosofía de D. Hume ha sido tradicionalmente vinculada con el positivismo y con el subjetivismo moral. Si bien es innegable su explicita influencia en estas escuelas de pensamiento, ello no obsta a la existencia efectiva de relaciones de armonía entre propuestas tradicionalmente opuestas a la filosofía humeana. En el presente trabajo se presentarán sus convergencias con la ética de la ley natural, particularmente la desarrollada por la llamada New Natural LawTheory. Se argumentará el vínculo a partir de (a) la importancia de la vida común, (b) de la experiencia y (c) del rol de la filosofía en el comportamiento humano.

PALABRAS CLAVE: Ley de Hume; experiencia; vida común; bienes básicos: subjetivismo.

\section{Relations between Hume's philosophy and Natural Law Ethics}

ABSTRACT: The philosophy of D. Hume has been commonly related to positivism and moral subjectivism. Though his explicit influence is undeniable in these schools of thought, it does not prevent the effective existence of relations of harmony between theories traditionally opposed to the humean philosophy. In this work I will present the convergences between the philosophy of Hume and the natural law ethics, particularly the developed by the New Natural Law Theory. I will argue the link from the following points: (a) the relevance of the common life, (b) the experience and (c) the role of philosophy in the human behavior.

KEY WORDS: Hume's law; experience; common life; basic goods; subjectivism.

\section{INTRODUCCIÓN}

La filosofía de David Hume ${ }^{1}$ ha sido tradicionalmente relacionada con filosofías de corte positivista y con éticas de carácter subjetivista. Así, J. L. Mackie $^{2}$ señala que su obra ética ${ }^{3}$ tiene como base y soporte en gran medida la filosofía de los moralistas ingleses, refiriéndose en particular a Hume. Por su parte, la epistemología empirista, junto con el rechazo a la metafísica entendida como «extralimitación cognoscitiva» ${ }^{4}$ ha identificado a Hume como uno de los grandes precursores del neopositivismo o «empirismo analítico» ${ }^{5}$.

" Doctor (c) en Filosofía, Pontificia Universidad Católica de Chile, Facultad de Filosofía. Profesor de Éticas Aplicadas, Pontificia Universidad Católica de Chile, Facultad de Filosofía. Docente de Lógica Jurídica, Universidad Bernardo OHiggins, Facultad de Ciencias Sociales, Departamento de Ciencias del Derecho.

1 En este trabajo se citaran abreviados los siguientes textos de Hume: (a) el Treatise of Human Nature como THN; (b) la Enquiry Concerning Human Understanding como EHU. Los pasajes en castellano del THN serán tomados de la traducción de Félix Duque.

2 Cf. MACKIE, 1980: viii.

3 Refiriéndose a MACKIE, 2000.

4 Cf. TASSET, 1999: 59-60.

5 Cf. Stroud, 1977: 219 y ss. 
Desde esta perspectiva, puede parecer forzado encontrar puntos de convergencia entre dos propuestas éticas que se inspiran en tradiciones opuestas. La ética de la ley natural, desde la exposición de sus representantes contemporáneos más destacados ${ }^{6}$, toma como punto de referencia la tradición aristotélico-tomista $^{7}$. La filosofía de Hume, por el contrario, se distancia de la tradición filosófica, la que encuentra entre sus representantes más paradigmáticos, a Aristóteles y al aquinate, los mismos en los cuales los teóricos de la ley natural dicen inspirarse.

Sin embargo, la visión tradicional de la filosofía humeana, junto con la caricatura frecuente de las que las teorías de la ley natural son objeto, no son la última palabra en cuanto se trata de encontrar elementos comunes. Si bien es cierto que ambas tradiciones parten de presupuestos distintos y opuestos, no es menos cierto que comparten ciertas intuiciones respecto de temas éticos y meta-éticos. No se intentará realizar en este trabajo una compatibilización a nivel de sus presupuestos teoréticos fundamentales, sino más bien, mostrar las convergencias desde la perspectiva de las intuiciones, las que encontrarán a nivel de los principios, distintos fundamentos según se trate de la filosofía humeana o de la teoría de la ley natural. Con «intuiciones» nos referimos a posicionamientos pre-teoréticos acerca de distintos temas, los cuales pueden tener -aunque, no necesariamente tendrán- un correlato en aspectos filosóficos sistemáticos. Por supuesto, dichas intuiciones podrán fundamentarse de modo muy distinto, y las diversas elaboraciones filosóficas de las mismas tendrán que pasar el test de la coherencia, entre otros. El punto que quisiéramos poner de relieve con esta compatibilidad a nivel de intuiciones es que, en último término, la elaboración filosófica tiene, en no pocos casos, un rol subordinado a las mismas, en la medida en que lo que se busca con estas elaboraciones es una fundamentación de intuiciones fundamentales, las cuales se encuentran allende la dialéctica filosófica. Esto, nuevamente, no obsta a que, naturalmente, de éstas confluencias intuitivas puedan surgir confluencias sistemáticas. Se mencionarán algunas de ellas en el último apartado de esta investigación

\section{La Ética de La Ley natural en CONTEXTO}

La teoría de la ley natural (o iusnaturalismo) es un planteamiento de ética, filosofía política y del derecho, cuyas fuentes clásicas son Aristóteles y Tomás de Aquino, entre los más importantes. Dicho planteamiento ha sido desarrollado profusamente en la historia de la filosofía, comenzando por el

\footnotetext{
6 Cf. Grisez, 1965, FinNis, 1980, GeORge, 1999.

7 Cabe hacer notar que un elemento de convergencia indirecto desde el punto de vista de la tradición que opera como referencia de dichas propuestas podría estar dado, al menos en el caso de Finnis y George, por el positivismo jurídico, que da el marco conceptual de la revitalización de la teoría de la ley natural como propuesta vigente en filosofía del derecho.
} 
iusnaturalismo tradicional, pasando por los autores de la escolástica tardía, la modernidad y la escuela de los derechos naturales (natural rights) hasta las actuales perspectivas de la denominada New Natural Law Theory. Desde la época moderna, la tradición clásica del derecho natural ha sido objeto del desprestigio generalizado que afectó a toda la filosofía de la tradición antigua y medieval, siendo Hume uno de los filósofos más críticos en este sentido, como veremos. En la modernidad, el iusnaturalismo muta hacia una consideración, no de la ley natural o del derecho natural (natural law) sino de los derechos naturales (natural rights), encontrándose entre ellos, los típicos que identifica el liberalismo clásico (libertad, propiedad). Esta interpretación liberal y moderna no tiene en principio parentesco genuino con la teoría clásica de la ley natural, si bien es cierto que en la literatura se puede observar una terminología que puede llevar a equívocos.

La actitud hostil con la tradición clásica del derecho natural en particular y con la escolástica en general se mantiene hasta mediados del siglo XX. En el ámbito de la filosofía del derecho, donde tradicionalmente se estudiaban, desde una perspectiva histórica, las ideas de Tomás de Aquino respecto de la ley, primó de modo indiscutido una visión iuspositivista, la que se puede resumir en la autonomía del derecho que estos autores reclaman frente a la moral. El iusnaturalismo del s. XX, que sostenía la correlación y mutua dependencia del derecho y la moral, aún delimitado por la tradición escolástica, no tenía los elementos teóricos suficientes para hacer frente a un iuspositivismo bastante desarrollado, a la luz de los aportes de John Austin ${ }^{8}$ y Hans Kelsen ${ }^{9}$.

A partir de la apertura de los iuspositivistas posteriores como H. L. A. Hart ${ }^{10}$ y J. Raz ${ }^{11}$ hacia las ciencias sociales, fue posible que, dentro del seno mismo de la tradición iusfilosofica positivista, surgiera la posibilidad de replantearse la teoría de la ley natural ${ }^{12}$. Es en este estado de la cuestión en que aparece la figura de John Finnis, discípulo de Hart, quien fue formado en la tradición del positivismo jurídico analítico, pero que, con posterioridad, fue profundizando en la tradición de la filosofía clásica que era en gran medida desconocida por sus contemporáneos, lo que demuestra a través del agudo análisis de las caricaturas de la ley natural que realiza en una de sus principales obras, Natural Law and Natural Rights ${ }^{13}$. Este último puede ser considerado como «uno de los autores que han servido de puente entre una teoría moral de inspiración clásica —aunque renovada y no exenta de polémica-y las filosofías jurídicas y morales contrarias, vigentes hoy en la mayor parte de los ambientes intelectuales de Europa y América» ${ }^{14}$.

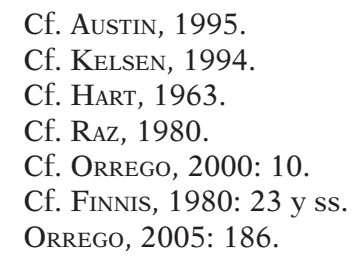


La escuela más representativa del iusnaturalismo contemporáneo es la llamada New Natural Law Theory. Si bien ella surge con el artículo de Grisez ${ }^{15}$ no es sino hasta la obra de Finnis ${ }^{16}$ en la que esta tradición recoge los elementos propios de la filosofía hartiana y, por lo tanto, se hace una alternativa atractiva a los distintos positivismos jurídicos en boga en ese tiempo.

Dos de los elementos fundamentales de la ética de esta escuela de pensamiento son los «valores o bienes básicos para el florecimiento humano» y los «principios de razonabilidad práctica». Para efectos explicativos, podemos definir a los primeros como formas fundamentales de bienestar humano, cuyo conocimiento está dado por la experiencia y que son caracterizados por ser inderivados, autoevidentes e inconmensurables, llevando todo lo anterior a la consideración de su objetividad ${ }^{17}$. En cuanto a los principios de razonabilidad práctica, podemos definirlos como ciertas exigencias metodológicas básicas para un buen juicio práctico, el cual toma como eje la consideración de los otros bienes o valores básicos para determinar y especificar la participación de una persona en uno o más de esos valores; considerados éstos, es posible aplicar dichas exigencias en determinados actos y proyectos para, así, generar juicios en torno a lo razonable o irrazonable de los $\operatorname{mismos}^{18}$. Como la moral se define en términos de razonabilidad práctica ${ }^{19}$, todo acto considerado razonable es, por consiguiente, un acto moralmente correcto, y todo acto irrazonable es a su vez, moralmente incorrecto. Es menester señalar que la razonabilidad práctica es considerara por estos autores, también, un valor básico ${ }^{20}$.

El vínculo entre las formulaciones clásicas de la ley natural con las expresiones actuales, puede explicarse del siguiente modo: «las exigencias a las que ahora atenderemos expresan "el método de la ley natural" para elaborar la "ley natural" (moral) a partir de los primeros "principios de la ley natural" (pre-morales)» ${ }^{21}$. En otras palabras: las exigencias de razonabilidad práctica expresan el método de la ley natural para elaborar unas pautas morales generales (la ley natural propiamente tal) a partir de los valores o bienes básicos del florecimiento y bienestar humano (esto es, los primeros principios de la ley natural).

\section{TRAdiciones Contrapuestas}

La tradición que abre Hume en la historia de la filosofía es el empirismo. Si bien es cierto no es posible reducir su pensamiento a la dimensión

\footnotetext{
15 Cf. Grisez, 1965.

16 Cf. FinNIS, 1980.

17 Cf. Finnis, 1980: 59 y ss.; Gómez-Lobo, 2002: 6 y ss.

18 Cf. Finnis, 1980: 100 y ss.; Gómez-Lobo, 2002: 41 y ss.

19 Cf. FinNIS, 1980: 15.

20 Cf. Finnis, 1980: 88; Gómez-Lobo, 2002: 20-21.

21 FinNIs, 1980: 103; traducción castellana de FinNIs, 2000: 134.
} 
epistemológica, ella sirve de base a toda su reflexión. Podemos definir a grandes rasgos el empirismo como aquella teoría epistemológica que sostiene que todo conocimiento posible deriva de la experiencia. Para nuestro autor, la experiencia está compuesta por dos elementos: ideas e impresiones ${ }^{22}$. Desde esa base, Hume y el empirismo que le sigue toman posición respecto de las diversas problemáticas, particularmente morales y políticas, las que, como han señalado varios autores, eran las principales preocupaciones del escocés ${ }^{23}$.

Podemos sintetizar el núcleo del empirismo humeano a partir de los siguientes principios ${ }^{24}$ : (i) la observación y la experiencia como el único fundamento viable y fiable del conocimiento humano ${ }^{25}$; (ii) el rechazo de los razonamientos hipotéticos, entendiendo por «hipótesis» todo principio o razonamiento que no encuentre su verificación a través de, y que no sea contrastable con, la experiencia; (iii) el rechazo de toda metafísica y de toda «superstición» aludiendo con este término tanto a la metafísica como a toda manifestación de carácter religioso, fundamentando el rechazo de ambas por la supuesta "extralimitación cognoscitiva» que suponen al no basar sus razonamientos en la experiencia; (iv) el carácter progresivo del conocimiento, en un abierto rechazo a la tradición racionalista, que consideraba como verdadero conocimiento aquel inmutable.

La ética de la ley natural, por su parte, tiene sus fuentes más clásicas en Aristóteles y Tomás de Aquino. Si bien Hume nunca lo explicita, pareciera que su filosofía surge como respuesta a una larga tradición que considera a éstos como sus autores paradigmáticos ${ }^{26}$. Es posible rastrear esta crítica en varios de los elementos más originales de la obra de nuestro autor: (i) la crítica del principio de causalidad; (ii) la crítica a la idea de sustancia; (iii) la idea de que la razón no pueden en ningún caso mover la voluntad. De este modo, la filosofía humeana parece posicionarse en abierta oposición a la tradición que fundamenta la ética de la ley natural.

Los elementos esenciales que toma la teoría de la ley natural de la tradición aristotélico-tomista se basan fundamentalmente en la caracterización que hace Aristóteles de la razón práctica, la que es retomada por Tomás de Aquino para complementarla con sus observaciones respecto de la naturaleza de la ley. Aristóteles distingue entre el intelecto especulativo y el intelecto práctico (noûs praktikós). Mientras que el primero está dirigido hacia la contemplación

\section{Cf. THN I, i, 1; EHU II.}

23 La concepción de Hume como «filosofo de la vida común» radica en la importancia que tiene para el hombre común y para la vida cotidiana del hombre estos temas (Cf. Livingstone, 1983; RÁBADE, 1998).

$24 \quad$ La siguiente síntesis es de TASSET (1999: 57 y ss.).

25 Como señala TASSET (1999: 59), las alusiones a «experimentos» en la obra de Hume deben entenderse como aludiendo a la experiencia.

26 Cf. THN, introducción. Ahí alude a «argumentos abstrusos», cuyos «principios asumidos confiadamente» han llevado al «descredito de la filosofía». 
y en último término, a la sabiduría (sophía), el segundo está dirigido hacia la acción, operando a través del denominado silogismo práctico ${ }^{27}$.

Este tema es elaborado por Aristóteles desde elementos comunes a animales y seres humanos, para posteriormente rescatar los elementos del silogismo práctico humano. En los animales, el problema de la acción es vinculado a la satisfacción de las necesidades básicas para el mantenimiento de la vida, mientras que en el caso del hombre «el movimiento de traslación y las posibilidades que éste abre están conectados no solo con la satisfacción de necesidades vitales inmediatas, sino también $-\mathrm{y}$ fundamentalmente- con la realización de un conjunto muy amplio de actividades encaminadas a la configuración de la propia vida de acuerdo con una cierta representación de lo que sería una vida buena y deseable para el hombre ${ }^{28}$. La conclusión de este silogismo práctico es una acción ${ }^{29}$.

En Aristóteles, la acción está determinada por dos elementos: el factor desiderativo y el factor cognitivo, siendo el primero el que determina el fin, mientras que el segundo juzga los medios y establece el fin como realizable o $n^{30}$. Distingue, respecto del deseo (órexis), varios tipos, siendo la boúlesis o «deseo racional», aquel vinculado "con los fines y expectativas de largo plazo que el agente humano asume como propias, en un cierto esbozo de lo que sería para él la vida buena y lograda ${ }^{31}$. La definición del carácter racional y por lo tanto, propiamente humano, de la acción, radica en que «en el caso del hombre, justamente en la medida en que a través de los deseos racionales tiene acceso a un horizonte de fines de mediano y largo plazo, puede surgir respecto de una situación particular de acción un conflicto motivacional entre dichos deseos racionales y los deseos apetitivos más inmediatos» ${ }^{32}$.

En este aspecto, la filosofía humeana toma como elemento motivante de la acción, sólo las pasiones. Como señala su famosa cita del Treatise: «la razón no puede ser nunca motivo de una acción de la voluntad [...] La razón es, y sólo debe ser, esclava de las pasiones, y no puede pretender otro oficio que el de servirlas y obedecerlas ${ }^{33}$. En este sentido, no solo se desmarca de la tradición escolástica, sino que también de la racionalista. Descartes, por ejemplo, considera a la naturaleza y también a las percepciones sensibles, pero su naturaleza es una naturaleza teologizada, a diferencia de la humeana, explícitamente desteologizada ${ }^{34}$; para Spinoza la naturaleza y la fuerza de los afectos está supeditada a la razón ${ }^{35}$.

27 Cf. De Motu Animalium, 6-7; De Incesso Animalium; De Anima, III, 7, 9-13 (en adelante DA).

28 VIGO, 2007: 110.

29 VIGO, 2007: 112; Ethica Nicomachea, VII, 5 (en adelante EN).

30 VIGO, 2007: 112.

31 VIGO, 2007: 113; Cf. EN III, 4; DA II, 3.

32 VIGO, 2007: 114.

33 THN, II, iii, 3: 415.

34 Cf. RÁBADE, 1998: 18.

35 Cf. RÁBADE, 1998: 19. 
Para este efecto, nuestro autor elabora una epistemología que explique adecuadamente la naturaleza de las pasiones y el sentimiento, ya que estas son las piezas fundamentales para explicar la naturaleza de la moral y en último término, la preocupación de Hume por la vida del hombre común ${ }^{36}$. En este sentido va la denominación de su propuesta como "gnoseología del sentimiento» ${ }^{37}$.

Nuestro autor define la pasión como una «existencia original»o también, una «modificación de existencia» ${ }^{38}$ y sostiene que no contiene ninguna «cualidad representativa» ${ }^{39}$. Por ello declara que "es imposible [...] que a esta pasión se puedan oponer la verdad y la razón, pues esta contradicción consiste en el desacuerdo de ideas, consideradas como copias, con los objetos que representan $»^{40}$. La única forma de oposición entre pasión y razón, declara Hume, se da «en cuanto que acompañadas de un juicio u opinión $»^{41}$.

A diferencia del pensamiento humeano, la tradición aristotélico-tomista entrega un rol destacado a la razón en la definición de la conducta y, en contraste con Hume, de delimitación del rol de las pasiones en la determinación de la acción. Para Aristóteles, deseo apetitivo o epithymía es una forma de deseo que es común tanto a hombres como a animales; sin embargo, en virtud de la capacidad propiamente racional del hombre, la epithymía debe ser conforme a una decisión deliberada o proaíresis, esto es, "un deseo deliberativamente mediado de aquello que aparece como realizable para el agente, por haber sido determinados exitosamente los medios que conducen a su obtención » ${ }^{42}$. A través de los deseos racionales, los seres humanos, están arrojados hacia un horizonte futuro de posibilidades, por lo que puede darse el caso que los deseos inmediatos confronten los deseos racionales de mediano y largo plazo en virtud de las consecuencias de los primeros para los segundos ${ }^{43}$. Para Aristóteles, los deseos racionales (boúlesis) tienen preeminencia respecto de los apetitivos (epithymía).

De modo similar Tomás de Aquino concibe la acción y el rol de la razón. Para él, la razón es el primer principio de los actos humanos ${ }^{44}$, operando como «regla y medida» de los mismos ${ }^{45}$. La ley, en este caso, es una prescripción de la razón ${ }^{46}$. La razón para Tomás y Aristóteles, y a diferencia de la filosofía humeana, mueve a obrar de modo preeminente.

De este modo, para sintetizar, la oposición respecto de los fundamentos tanto de la filosofía humeana como del background de la ética de la ley natural,

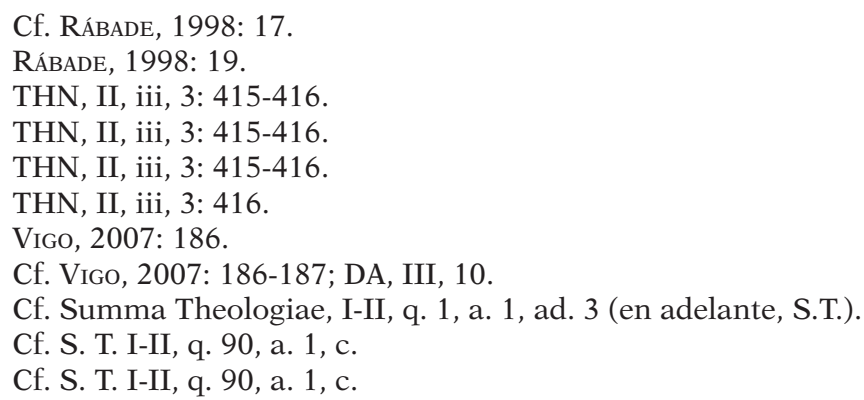


radica, no solo en las ya bastante conocidas críticas de Hume a los elementos más clásicos de la metafísica occidental, tales como la teoría de la sustancia y de la causalidad, y de la posibilidad misma de la metafísica, sino también respecto de la concepción de la acción y en particular, del lugar de la razón para motivar y determinar la misma. Mientras que para la tradición aristotélicotomista, el motor fundamental de la acción es la razón, para Hume es la pasión.

\section{ReLACIONES}

\section{a. La «ley de Hume»}

Desde el renovado interés que desde el siglo pasado se despertó por la filosofía de Hume, se han establecido una serie de relaciones entre ella y la ética de la ley natural. La principal relación se ha establecido entre un pasaje del Treatise, en el que nuestro autor propone lo que ha sido llamado posteriormente la «Ley de Hume», y que ha sido tradicionalmente vinculado con la denominada «falacia naturalista» o el paso indebido del ser al deber-ser. El pasaje en cuestión es el siguiente:

En todo sistema moral de que haya tenido noticia, hasta ahora, he podido siempre observar que el autor siegue durante cierto tiempo el modo de hablar ordinario, estableciendo la existencia de Dios o realizando observaciones sobre los quehaceres humanos, y, de pronto, me encuentro con la sorpresa de que, en vez de las copulas habituales de las proposiciones; es y no es, no veo ninguna proposición que no esté conectada con un debe o un no debe. Este cambio es imperceptible, pero resulta, sin embargo, de la mayor importancia. En efecto, en cuanto que este debe o no debe expresa una nueva relación o afirmación, es necesario que esta sea observada y explicada y que al mismo tiempo se de razón de algo que parece absolutamente inconcebible, a saber: cómo es posible que esta nueva relación se deduzca de otras totalmente diferentes ${ }^{47}$.

Este pasaje ha sido diversamente interpretado por la tradición filosófica. En general puede considerarse como el antecedente de la formulación que hace Moore de la denominada "falacia naturalista ${ }^{48}$, que puede entenderse como la asociación o la confusión del término «bueno» con una propiedad natural como, por ejemplo, el rojo.

47 «In every system of morality, which I have hitherto met with, I have always remark'd, that the author proceeds for some time in the ordinary way of reasoning, and establishes the being of a God, or makes observations concerning human affairs; when of a sudden I am surpriz'd to find, that instead of the usual copulations of propositions, is, and is not, I meet with no proposition that is not connected with an ought, or an ought not. This change is imperceptible; but is, however, of the last consequence. For as this ought, or ought not, expresses some new relation or affirmation, 'tis necessary that it shou'd be observ'd and explain'd; and at the same time that a reason should be given, for what seems altogether inconceivable, how this new relation can be a deduction from others, which are entirely different from it». THN III, i, 1: 47.

48 Cf. Moore, 1903: par. 12. 
Finnis sostiene que la llamada «Ley de Hume», asociada con la «falacia naturalista» es uno de los principales argumentos en contra de la existencia de una «ley natural $»^{49}$. Sin embargo, también señala, respecto de la derivación de normas respecto de hechos, que los teóricos de la ley natural «no lo han hecho, no necesitan hacerlo, ni los exponentes clásicos de la teoría soñaron con intentar alguna derivación de este tipo» ${ }^{50}$. El enfoque de Finnis a este respecto, enfoque que por lo demás es compartido por toda la New Natural Law Theory, puede ser denominado como enfoque epistemológico. Los "primeros principios de la ley natural», esto es, los valores o bienes básicos para el florecimiento humano, son principios autoevidentes de la razón práctica, y no necesitan ser derivados de una explicación metafísica de la naturaleza humana ${ }^{51}$. Este elemento, no obstante, ha generado controversia dentro de la misma teoría iusnaturalista, más allá del núcleo de la New Natural Law Theory. Es posible definir esta problemática como la aceptación o el rechazo de la naturaleza como criterio de moralidad ${ }^{52}$.

Podemos señalar, en el contexto de esta discusión que, más allá de la efectiva reconciliación que es posible realizar en el núcleo del iusnaturalismo contemporáneo, particularmente el de la tradición analítica, entre razón práctica y naturaleza como criterio de moralidad $^{53}$, es innegable que la aceptación de la «Ley de Hume» y la «falacia naturalista» como argumento válido por parte de esta tradición revela una profunda influencia de Hume en la totalidad del pensamiento analítico, incluso en aquella que puede considerarse como deudora de la filosofía clásica. No en vano Finnis dedica varias páginas a analizar la «Ley de Hume» ${ }^{54}$. La aceptación de la misma, a tono con casi toda la filosofía moral analítica en boga en ese momento, le lleva a plantear el problema en términos de razonabilidad práctica, o en terminología escolástica, un derecho natural «secundum rationem», en oposición a uno «secundum naturam» ${ }^{55}$.

\section{b. Subjetivismo vs. realismo moral}

Es necesario, finalmente, señalar las relaciones de la ética de la ley natural con éticas de inspiración humeana. Aquí cabe indicar a dos autores ya mencionados en este trabajo: John Mackie y John Finnis. El primero, en su Ethics: inventing right and wrong ${ }^{56}$, profundizaa en el subjetivismo moral, el cual

\footnotetext{
49 Cf. FinNis, 1980: 33 y ss.

50 Cf. FinNIS, 1980: 33; traducción castellana de Finnis, 2000: 66.

51 Cf. FINNIS, 1980: 33-34.

52 Cf. GONZÁLEZ, 2006: 37-38.

53 CF. George, 1992: 31 y ss.

54 Cf. FinNIS, 1980: 36 y ss.

55 Cf. GonZÁLEZ, 2006: 40.

56 Cf. MACKIE, 2000.
} 
—posteriormente indicara - tiene mucho de inspiración humeana ${ }^{57}$; el segundo, en su Fundamentals of Ethics ${ }^{58}$, penetra en el realismo moral ya desarrollado en su Natural Law and Natural Rights.

En el contexto de su obra de 1983, Finnis polemiza con Mackie, sosteniendo que los dos argumentos principales para el escepticisimo moral son aquellos que el autor subjetivista denomina el argumento «de la relatividad» y el argumento «de la singularidad» (queerness) ${ }^{59}$. El primero sostiene, en primer lugar, la variación de los códigos morales entre una y otra sociedad o época y «las diferencias entre los credos morales de los distintos grupos de una sociedad compleja $»^{60}$, que si bien considera un hecho de moral descriptiva, «puede proporcionar un apoyo indirecto para el subjetivismo de segundo orden: las diferencias radicales entre los juicios morales de primer orden dificulta el que estos puedan ser tratados como aprehensiones de verdades objetivas» ${ }^{61}$. El argumento de la singularidad o «queerness» — sostiene Mackie— consta de dos partes, una metafísica y una epistemológica. En cuanto a la primera, señala que «si hubiera valores objetivos, debería haber entidades de muy extraña naturaleza, enteramente distintas de cualquier entidad del universo» ${ }^{62}$. En cuanto a la segunda, «si fuésemos conscientes de ellos — los valores objetivosdebería existir una peculiar facultad de percepción moral o de la intuición que fuera absolutamente dispar con respecto a nuestra manera común de conocer las otras cosas» ${ }^{63}$.

La aproximación que realiza Finnis a estas consideraciones consiste, en primer lugar, en revisar la concepción de verdad y objetividad que subyacen al subjetivismo. Sostiene — citando a Mackie- que, para el escepticismo moral, «las supuestas cualidades objetivas de los actos, estados de cosas, etc., son realmente solo la proyección de sentimientos y deseos» ${ }^{64}$. Afirma, luego, que «las intenciones son completamente diferentes de cualquier otra cosa en el universo ${ }^{65}$, concluyendo, contra el argumento de la singularidad, que:

Si uno toma como modelo de entidades, cualidades y relaciones solo las entidades, cualidades y relaciones que figuran en las teorías físicas, químicas, bioquímicas... (y si no se realiza ninguna pregunta acerca de qué significa ser una teoría y para una teoría ser verdadera) entonces, se estaría inclinado a sostener que intenciones, significados y la verdad son completamente singulares $(q u e e r)^{66}$.

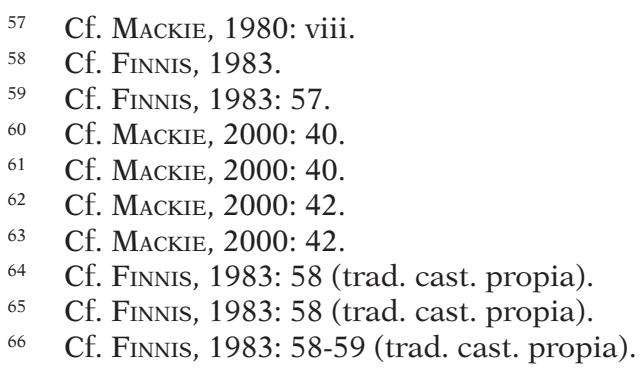


En otras palabras: Finnis sostiene que en la calificación de «singulares» (o «raros» desde una traducción literal de la palabra inglesa queer) que se les da a los valores objetivos hay una serie de presupuestos que también pueden ser aplicados en contra de la misma explicación de Mackie. En efecto, los sentimientos y deseos no pueden ser analizados desde una perspectiva empirista a menos que sean considerados supuestos inanalizables, primitivos o, en último término, comprometerse con una explicación reductivista: todos los fenómenos de la conciencia y de la voluntad pueden ser reducidos a procesos físico-químicos, los cuales pueden ser descritos adecuadamente por la ciencia natural.

\section{Confluencias}

Como hemos visto, la ética de la ley natural en sus manifestaciones contemporáneas tiene varias y variadas relaciones con la filosofía humeana, si bien es cierto hay una relativa oposición en sus presupuestos fundamentales. Parte de estas relaciones se dan tanto por una aceptación de algunas tesis del autor escocés (como por ejemplo, la «ley» que lleva su nombre) así como también el debate suscitado entre el subjetivismo moral de inspiración humeana con el realismo moral de la escuela iusnaturalista.

Sin embargo, es posible establecer un nivel aun mayor de confluencia. No solo el iusnaturalismo contemporáneo, y en concreto el de la New Natural Law Theory, ha aceptado la Ley de Hume, sino que también es posible rastrear confluencias a nivel de intuiciones fundamentales. Estas tienen que ver con la valoración de la vida común, con el rescate de la experiencia como criterio de aprendizaje moral y finalmente, el rol de la filosofía para comprender, aclarar y motivar la acción del hombre en su vida (común). Si bien todos estos elementos pueden ser rastreados hasta Aristóteles y Tomás de Aquino, han vuelto a adquirir relevancia a partir de los nuevos desarrollos del iusnaturalismo.

\section{a. Valores básicos, autoevidencia y vida corriente}

En primer lugar, podemos señalar la relación entre la autoevidencia que se le atribuye a los bienes básicos, con la consideración de la vida corriente como el marco desde el cual la moralidad es analizada. Esta vida común y/o corriente, Hume la entiende como «el curso ordinario de nuestro vivir, como la serie de ocupaciones en que empleamos nuestro tiempo en el fuero privado, en las relaciones sociales, en el mundo de los negocios, en el disfrute de los placeres ${ }^{67}$. Es en estas mismas circunstancias en que la problemática moral, la pregunta por lo correcto o incorrecto, se hace presente. Para Hume, «las decisiones filosóficas no son otra cosa que las reflexiones sobre la vida ordinaria,

67 RÁBADE, 1998: 16. 
tratadas con método y corrección ${ }^{68}$. Para nuestro autor, la filosofía se anclaría en la vida cotidiana y su rol no es otro que el de aclarar la «lógica» que subyace detrás de ella y de los juicios que hacemos en el devenir de ésta.

Dos maneras de establecer la relación entre esta idea y la ética de la ley natural son posibles: la primera radica en el carácter práctico de la ética que afirman estos autores; la segunda, a partir de la noción de autoevidencia de los bienes básicos y su vínculo con la noción de razón pública ${ }^{69}$.

Respecto de la primera idea, los autores iusnaturalistas como Finnis hacen hincapié en la idea clásica de que la ética es un conocimiento esencialmente práctico $^{70}$, en cuanto es un conocimiento que tiende hacia la acción y hacia la transformación personal. En el caso de Aristóteles, esto se manifiesta en el desarrollo de una ética de la virtud; en el caso de los autores iusnaturalistas, en la prosecución de ciertos bienes básicos que constituyen la realización humana fundamental.

La segunda idea es más compleja, y se refiere a la relación que hace el iusnaturalista Christopher Wolfe entre la autoevidencia de los bienes humanos básicos con el concepto de razón pública. Si bien el contexto de esta discusión es diferente de nuestro tema, hay elementos en ella para fundar una intuición común entre la filosofía humeana y el iusnaturalismo.

John Rawls sugirió el concepto de razón pública para distinguir los discursos que pueden tener cabida en la discusión pública, estableciendo una serie de requisitos para los mismos. Los enunciados realizados en el foro público deben ser: (i) realizados públicamente; (ii) debatidos abiertamente y (iii) aceptados ampliamente. Esto implica, por el contario, que debemos abstenernos de dar razones que (i) sean privadas; (ii) que sean demasiado complejas o demasiado amplias para ser entendidas y que (ii) no puedan ser entendidas por personas razonables ${ }^{71}$.

Wolfe critica la idea de razón pública dado que las exigencias anteriores pueden implicar que el ámbito de las personas razonables sea muy limitado. Por ello afirma que:

Si todos los ciudadanos tienen que entenderla, la razón pública liberal está en problemas, porque es difícil imaginar muchos argumentos que todos los ciudadanos entenderían. Algunas personas no son muy inteligentes y muchas otras no han sido bien educadas ${ }^{72}$.

De este modo, el conocimiento o la realización de lo correcto o incorrecto no pueden depender de las condiciones intelectuales de una persona. Sin embargo, ha sido un lugar común atribuir al iusnaturalismo una visión excesivamente racionalista de la moral. Tal es la crítica de los autores liberales que está

\footnotetext{
68 RÁBADE, 1998: 16

69 Cf. Wolfe, 2006. También: Arancibia, 2017: cap. 83-7.

70 Cf. Finnis 1983, cap. 1.

71 Cf. Wolfe, 2006: 28-9; Arancibia, 2017: 84.

72 Wolfe, 2006: 29 (trad. cast. propia)
} 
enfrentando Wolfe. Según Macedo - uno de los autores recientes de esta tradición liberal- la ética de la ley natural falla en cumplir los requisitos de la razón pública, porque, a su juicio, el derecho natural exige un proceso de inferencia moral que «requiere una sabiduría o razonabilidad no encontrada en ninguna persona o al menos en la mayoría. Entonces, insistir en dichos argumentos es contrario a la equidad de respeto encarnados en los cánones liberales de la justificación pública ${ }^{73}$. Wolfe contesta señalando que no es efectivo que la teoría de la ley natural requiera este proceso de inferencia análogo al que podría existir en lógica entre las normas morales concretas y los primeros principios prácticos (mediados, claro está, por los principios de razonabilidad práctica). En segundo lugar, tampoco es necesario algún tipo de inteligencia especial para el conocimiento o la captación de dichos principios y normas. Los teóricos contemporáneos han señalado que dichos principios son autoevidentes. «Las normas morales más generales son normalmente sabidas por todos, pero pueden ser borradas por persuasiones malvadas y por costumbres viciosas y hábitos corruptos [...] Esto es, mucha gente puede saber que algo está mal, pero no necesariamente ser capaz de articular ese conocimiento muy bien ${ }^{74}$.

El argumento de Wolfe defiende la capacidad de gente normal de poseer conocimiento moral, al menos en un grado suficiente como para desenvolverse en la vida cotidiana. Lo que la gente, probablemente, no pueda hacer, es explicar filosóficamente dicho conocimiento. Es decir, las personas en general poseen conocimiento moral, aunque este conocimiento no necesariamente vaya aparejado a su fundamentación ${ }^{75}$.

Lo anterior pone de relieve que, para la ética de la ley natural, la acción humana no depende en ningún caso de elaborados razonamientos, sino de una actitud natural del hombre. Si bien es cierto que esta discusión nace dentro de la tradición liberal, muy preocupada por la razón de lo público, desde el iusnaturalismo se afirma que no es posible escindir al hombre mismo en dos dimensiones: la pública y la privada ${ }^{76}$. Esta intuición, creemos, es compartida por

73 MAcEdo, citado por Wolfe, 2006: 31.

74 Wolfe, 2006: 31; cf. Arancibia, 2017: 85-6.

75 Señala a este respecto: «Now the question that must be posed is this: when advocates of public reason contend that liberalism requires reasoned arguments that are or can be "widely accepted," arguments that "should be acceptable to everyone concerned," is it sufficient that arguments be "acceptable» to people on the basis of what I have called «inarticulate knowledge»? / If the answer is "no» - that is, if more developed and articulate knowledge is required - then I believe a political theory's requirement of public reason is self-destructive, because it is a demand for something that is hopelessly utopian. If the answer is «yes» - that is, if arguments can be considered publicly accessible even if they can be accepted only on the basis of inarticulate knowledge - then natural law arguments meet that (lower) standard of public accessibility. In fact, it is one of the great strengths of natural law theory that it can account for the fact that people in general, not just intellectual elites, are able genuinely to know fundamental moral principles - being «reasonable» in that sense - even when (as is often the case) they are not capable of much in the way of rational arguments for particular ethical positions (especially as the questions get more complicated)» (WoLFE, 2006: 32-33).

76 Cf. Arancibia, 2017: 77-81. 
la filosofía humeana, puesto que la razón de la vida corriente no puede escindirse de la razón de lo público. Es precisamente en ese ámbito de vida privada y social donde el verdadero ethos del hombre se revela. Es en las circunstancias cotidianas de la vida, y no en una razón pública abstracta, en el que, para la ética de la ley natural, radica la experiencia de los bienes humanos básicos.

Respecto del primer enfoque - el más general, ligado al carácter práctico de la ética- existe un vínculo sistemático en torno a la prioridad epistemológica de la experiencia. Para Hume, como vimos, es la vida cotidiana o corriente la que tiene la prioridad epistemológica, ya que la filosofía es una reflexión acerca de la «lógica» que subyace a nuestras decisiones cotidianas. Del mismo modo, los autores iusnaturalistas contemporáneos de la línea de Finnis han planteado claramente esta prioridad. Así, para este autor, es la ética la que tiene prioridad respecto de la metafísica en el conocimiento del hombre ${ }^{77}$. Es a través del conocimiento de las capacidades del ser humano como se conoce al ser humano desde una perspectiva metafísica. Por ello, para ambas filosofías, aquello que sea el hombre es, epistemológicamente, posterior a la experiencia humana más cotidiana; y si el conocimiento acerca de qué sea el hombre es posible, lo será a partir de un conocimiento práctico acerca de cómo debe el hombre actuar.

\section{b. Experiencia como criterio de discernimiento práctico}

De acuerdo a lo dicho anteriormente ya hemos adelantado una segunda intuición compartida por la filosofía humeana y la ética de la ley natural: la importancia de la experiencia. Para Hume, no podemos ir más allá de la experiencia en lo relativo a la ciencia del hombre ${ }^{78}$. «La ciencia de la que debe partir el filosofo es, por un lado, la procedente de la introspección y, por otro - y más importante- la procedente de la observación de la vida y la conducta humanas ${ }^{79}$. La experiencia les da, tanto al filósofo como al hombre corriente, el material desde el cual él puede observar su propia disposición hacia ciertas conductas y también la conducta de los otros.

Para el iusnaturalismo, la experiencia es fundamental, a la hora de tener un «acceso» a los bienes humanos básicos. Estos no son adquiridos a través de un razonamiento abstracto. Uno puede abstraer de diversas situaciones, a través del pensar filosófico, que ellas comportan o encierran la realización de uno o más bienes básicos. Pero ello no implica que estos sean abstracciones. Porque los bienes básicos son oportunidades abiertas y es en la vida cotidiana, en su devenir, en sus decisiones, en sus actos particulares, donde estos se hacen evidentes.

Hume «está instalado en la herencia filosófica del nominalismo de lo singular» ${ }^{80}$. Por ello rehúye de todo tipo de abstracciones y de razonamientos

\footnotetext{
77 Cf. FinNIS, 1983: 21.

78 Cf. THN, introducción.

79 TASSET, 1999: 54.

80 RÁBADE, 1998: 12.
} 
apriorísticos. La tradición de la ley natural, por el contrario, no puede decirse que haya sido influido sustancialmente por la tradición nominalista; sin embargo, es posible ver en su caracterización de los bienes básicos ciertos elementos de nominalismo, en la medida en que los bienes humanos no son abstracciones, no son entidades universales, sino que son esencialmente particulares, porque corresponden de modo particularísimo a cada persona. La filosofía es la que, por motivos de estudio y comprensión, puede «elevarlos» a categorías universales. Pero ello no implica un compromiso ontológico con entidades universales, que es muy distinto de señalar — como lo hace la teoría de la ley natural, y lo que la haría, a mi juicio, compartir, con Hume, cierta inspiración nominalista ${ }^{81}$ - que todos estos bienes se corresponden con cada persona en concreto. De este modo, respecto de la comprensión que esta tradición tiene de los bienes humanos, puede decirse que el iusnaturalismo es nominalista respecto de ellos, y en este sentido, comparte con la filosofía humeana una predilección por lo particular.

Un segundo vínculo entre el valor de la experiencia en la filosofía humeana como en la de la ley natural radica en la comprensión de la misma que esta última tiene, particularmente por influencia de Aristóteles y la noción de phronesis. El concepto de «razonabilidad práctica», ya explicado, tiene una inspiración en el término griego antedicho. Aristóteles la define como «un modo de ser racional verdadero y práctico, respecto de lo que es bueno y malo para el hombre ${ }^{82}$. Esta virtud intelectual está presente de modo más pleno en las "personas con mayor experiencia». Como señala Aristóteles: «uno debe hacer caso de las aseveraciones y opiniones de los experimentados, ancianos y prudentes no menos que de las demostraciones, pues ellos ven rectamente porque poseen la visión de la experiencia» ${ }^{83}$. La phronesis, en cuanto virtud intelectual, se ejercita a través de la experiencia. Tal es el modo en que es comprendida la «razonabilidad práctica» en los iusnaturalistas contemporáneos.

Finalmente, tanto para la ética de la ley natural como para la filosofía humeana, la filosofía adquiere su sentido en la medida en que cumple un rol en la vida humana concreta. Para Hume, «como para todo ser humano, su tarea fundamental es vivir, y la filosofía [...] si no se inserta en este vivir para comprenderlo y aclararlo, está errando su camino» ${ }^{84}$. La filosofía moral humeana - y esto, según Hume, vale para toda la filosofía- está dirigida a la acción y su rol es comprender y dirigir la conducta humana ${ }^{85}$.

La ética de la ley natural, por su parte, reconoce la distinción entre intelecto teórico e intelecto práctico, recalcando que la diferencia entre ambos es

81 Al menos desde la comprensión que tiene la filosofía contemporánea respecto del nominalismo. Sobre la discusión contemporánea, con énfasis en el nominalismo, véase Loux, 2006: 46 y ss.

82 Et. Nic. VI, 5: 1140b 5-6.

83 Et. Nic. VI, 11, 1143b 11-15.

84 RÁBADE, 1998: 12.

85 Cf. TASSET, 1999: 64. 
meramente operacional ${ }^{86}$. Sin embargo, esta misma tradición, que reconoce - con Hume - la diferencia que existe entre el es y el debe, plantea, en este mismo sentido, la autonomía de la reflexión moral respecto de consideraciones metafísicas: «epistemológicamente, (el conocimiento de) la naturaleza humana no es "la base de la ética"; más bien, la ética es un preámbulo indispensable para un conocimiento completo y profundo de la naturaleza humana» ${ }^{87}$. En palabras de Finnis, para el conocimiento de la «naturaleza humana» en su dimensión propiamente humana, no nos es posible comenzar reflexionando meramente desde supuestos metafísicos; es necesario remitirse al ámbito de la acción.

\section{c. Algunos comentarios sobre las confluencias sistemáticas de ambos planteamientos}

Mencionamos al inicio de esta investigación que no era su objeto buscar confluencias a nivel de principios respecto de los planteamientos humeano y iusnaturalista, ya que éstos han sido tradicionalmente considerados opuestos. Indicamos, también, que junto con buscar relaciones (ya sea convergentes o divergentes) entre estos dos planteamientos quisimos, especialmente, plantear convergencias a nivel de intuiciones fundamentales. Con intuiciones fundamentales nos referimos a aspectos pre-teoréticos de ambos planteamientos, cuyos desarrollos filosóficos constituyen los aspectos sistemáticos de ambas teorías. Con «sistemático» nos referimos, esencialmente, a los aspectos propiamente filosóficos que estructuran ambas teorías y que las constituyen a cada una como un sistema -más o menos-coherente.

En lo que sigue quisiéramos poner de relieve algunas posibles confluencias a nivel sistemático. Dichas confluencias no necesariamente deben obedecer a las mismas premisas. Bien pueden obedecer a premisas diferentes, siempre que ninguno de estos aspectos teóricos confluyentes sean contradictorios con, o estén excluidos de, estos aspectos teóricos más fundamentales que les sirven de premisa y que asumimos, a manera de hipótesis, como opuestos.

En primer lugar, es claro que Hume es un pensador nominalista. Dicho nominalismo, sin embargo, no es incompatible, desde el punto de vista sistemático, con la teoría de la ley natural. En efecto, como ya mencionamos en el apartado anterior, la exigencia de que los bienes básicos, como formas fundamentales de bien, sean relativas al agente concreto, puede integrarse en la línea del nominalismo de lo singular de Hume. Si bien podemos hablar, desde la ley natural, del bien de la vida o del conocimiento, dichas referencias son abstracciones de los bienes humanos concretos de las personas. Así, podemos hablar, literalmente, de $m i$ bien, de $m i$ vida, de $m i$ conocimiento, de la vida o el conocimiento del Papa Francisco, o del bien de la amistad en el lector de este artículo, pero no es necesario que tengamos, por ello, que hablar de la vida o del

86 Cf. FinNis, 1983: 11-13.

87 FINNIS, 1983: 21 (trad. cast. propia). 
conocimiento como bienes abstractos desde el punto de vista del compromiso ontológico. El teórico de la ley natural está comprometido ontológicamente con el bien concreto, pero no es seguro que deba estarlo respecto del bien abstracto. Esto puede comprenderse mejor en el contexto de los desarrollos recientes en metafísica analítica de propiedades ${ }^{88}$. Simplificando, en esta discusión están en juego dos posiciones fundamentales: el realismo, que plantea la existencia objetiva de propiedades entendidas como entidades abstractas universales, y el nominalismo, que niega la existencia de dichas propiedades, haciendo un análisis reduccionista de las mismas ${ }^{89}$. Así, el nominalista no está comprometido ontológicamente con la existencia de propiedades, si bien acepta que se hable de ellas, siempre y cuando este discurso no sea literal. El teórico de la ley natural, entonces, está comprometido ontológicamente con la existencia del bien concreto, y puede dar un análisis reduccionista del bien abstracto en la medida en que éste se refiera al bien concreto de los agentes morales.

En segundo lugar, es posible plantear confluencias sistemáticas a nivel metaético. La meta-ética es aquella reflexión filosófica que versa sobre los fundamentos de la ética, particularmente aquellos relativos a su fundamentación metafísica y epistemológica ${ }^{90}$. En general se ha entendido que Hume se enmarca dentro del nocognitivismo. Dicha doctrina afirma que los enunciados morales no constituyen creencias, sino una proyección de otro tipo de estados, como sentimientos ${ }^{91}$. En este sentido, Hume parece ser, indudablemente, un no-cognitivista.

Sin embargo, como ha puesto de relieve Sturgeon, la interpretación de la meta-ética de Hume dista mucho de ser sencilla. Existen varios aspectos de la obra de Hume que no son consistentes entre sí, aunque - afirma- es, en general, más consistente de lo que los intérpretes de su obra han propuesto ${ }^{92}$. Plantea, respecto su supuesto no-cognitivismo, lo siguiente:

Lo que complica la interpretación de Hume acerca de los juicios morales [...] es la existencia, tanto en el Tratado de la Naturaleza Humana como en la Investigación sobre los Principios de la Moral, de pasajes que aparecen como afirmaciones definitivas de una perspectiva que no es en absoluto no-cognitivista. Es, de seguro, una perspectiva que da a nuestros sentimientos morales un rol central en la evaluación moral, pero ese rol ha de ser el asunto de esas evaluaciones, aquello de lo que versan; y permiten que esas evaluaciones sean verdaderas o falsas ${ }^{93}$.

88 Una síntesis de este debate puede encontrarse en Loux, 2006.

89 Un autor realista fundamental es David Armstrong, 1978a, 1978b, 1983, 1989. Autores nominalistas relevantes son David Lewis, 1986 y Gonzalo Rodríguez-Pereyra, 2002.

90 Una buena introducción a la meta-ética es la de Miller, 2003.

91 Cf. Miller, 2003: 6.

92 Cf. Sturgeon, 2008: 513.

93 "What complicates the interpretation of Hume's view about moral judgments, despite the existence of these and some other passages we shall examine, is the existence, both in his Treatise of Human Nature and in the Enquiry concerning the Principles of Morals, of passages that look like definitive assertions of a different sort of view that is not noncognitivist at all. It is, to be sure, a view that gives our moral sentiments a central role in moral assessments, 
Lo anterior implica que los fundamentos de la ética de Hume no son, como podría pensarse, meramente proyectivistas (en el sentido de proyectar un estado sentimental) sino que constituyen creencias sobre dichos estados. Por lo tanto, la ética de Hume no puede ser no-cognitivista. En este punto, hay una confluencia sistemática importante respecto de la ética de la ley natural, que es una ética cognitivista.

En el mismo ámbito meta-ético, es posible, además, afirmar que Hume podría afirmar una ética realista. Si bien es cierto que esta ética es subjetivista (en el sentido de que los hechos morales están determinados por estados subjetivos de los agentes morales $)^{94}$ hay, sin embargo, un compromiso ontológico por hechos morales. Desde esta perspectiva, la ética humeana podría tener aún mayor confluencia con la ética iusnaturalista.

Lo anterior ha querido poner de relieve que el nivel de confluencia entre estas dos aproximaciones éticas puede, además, manifestarse en semejanzas a nivel sistemático. Por supuesto, la ética normativa que ambos planteamientos afirman dista mucho de ser similar al nivel de sus principios prácticos fundamentales como a nivel de las normas morales más usuales que dichas teorías reconocen. Sin embargo, esto no obsta a que estas intuiciones generales impliquen semejanzas sistemáticas. Por lo demás — como lo muestra el trabajo de Sturgeon- la interpretación de la meta-ética humeana dicha mucho de estar cerrada a nuevas interpretaciones.

\section{ConcLusiones}

En el presente trabajo quisimos recalcar algunos de los elementos que, a nuestro juicio, relacionan tanto la filosofía de Hume como el iusnaturalismo, con especial énfasis en los elementos compartidos. Estas semejanzas se hacen ver, en particular, en el análisis de los desarrollos actuales de la teoría de la ley natural, particularmente en la desarrollada por la New Natural Law Theory. Revisamos los distintos supuestos con los que operan tanto la filosofía humeana como el iusnaturalismo, para concluir la incompatibilidad de ambos a la luz de los elementos fundantes esenciales. Sin embargo, recalcamos que nuestra intención no era encontrar puntos en común a este nivel sino en ciertas formas de tratar temáticas relativas a la vida humana corriente, la experiencia y el rol de la filosofía. A nivel del tratamiento filosófico concreto, se nos hacían evidentes una serie de semejanzas que era necesario explicitar.

Comenzamos la relación entre ambas propuestas filosóficas a través de puntos de encuentro que han sido generalmente puestos de relieve por filósofos

but that role is to be the topic of those assessments, what they are about; and it allows these assessments to be true or false.» (STURGEON, 2008: 514). Los pasajes en cuestión que menciona Sturgeon son THN, III, ii, 1: 477 y el Enquiry Concerning the Principles of Morals (EPM), Apéndice 1: 107, entre otros.

94 Ésta es, precisamente, la lectura de Sturgeon, 2008: 514. 
e investigadores, tales como la relación entre el iusnaturalismo y la «Ley de Hume» y la "falacia naturalista», como así también la influencia humeana en filosofías morales de corte subjetivista, en oposición al realismo moral propio de las teorías iusnaturalistas. Luego, revisamos los puntos de encuentro a nivel del abordaje filosófico de ambas teorías respecto de problemáticas comunes. Ahí observamos varias semejanzas.

En primer lugar, encontramos una gran semejanza a la valoración de la vida común y corriente, particularmente en su dimensión moral. Mencionamos el enfoque de ambos planteamientos en torno al carácter práctico de la ética y la filosofía. Analizamos las críticas del liberalismo contemporáneo a la teoría de la ley natural en lo relativo a la razón pública, y vimos cómo estas críticas pueden ser enfrentadas para poner de relieve la actitud natural del hombre frente a la moral y a la razón. Señalamos que esta defensa muestra una semejanza entre la filosofía humeana y el iusnaturalismo, particularmente en cuanto es en la vida corriente, "privada», en donde los problemas morales son enfrentados y que, por lo mismo, la distinción entre lo «público» y lo «privado» no tiene sentido desde esta perspectiva, común a humeanos y iusnaturalistas.

En segundo lugar, indicamos las semejanzas entre ambos planteamientos respecto de la noción de experiencia. Dijimos que puede encontrarse un punto de unión entre humeanos y iusnaturalistas respecto del nominalismo, siendo en los primeros explícito y en los segundos, presunto. Los bienes básicos, en consonancia con este pretendido nominalismo, no son abstracciones o entidades universales, sino más, bien oportunidades abiertas que están radicadas en personas concretas y no en una «idea de hombre». En este mismo sentido vimos los vínculos que existen entre el concepto de «razonabilidad práctica» y la vocación humeana por la experiencia. Indicamos que la experiencia es fundamental para un "acceso» a los bienes humanos y que, en la experiencia, éstos se nos hacen evidentes.

En tercer lugar, indicamos una relación entre ambos planteamientos respecto de la concepción de la filosofía. Para ambos, la filosofía debe estar dirigida a la acción. En el caso del iusnaturalismo, al menos, la ética es esencialmente práctica y debe ser autónoma respecto de (más no independiente de) consideraciones metafísicas.

Finalmente, aún cuando supusimos por hipótesis la incompatibilidad en las premisas fundamentales de ambos sistemas, es posible encontrar semejanzas sistemáticas entre la filosofía de Hume y la ética de la ley natural. La interpretación de la meta-ética humeana dista mucho de estar cerrada a nuevas lecturas y, siguiendo a Sturgeon, planteamos la posibilidad de interpretar a autor inglés en clave cognitivista y realista. Lo anterior reflejaría una gran convergencia en los dos planteamientos estudiados ${ }^{95}$.

95 Esta investigación se realizó en el contexto de una beca para estudios de doctorado financiada por la Comisión Nacional de Ciencia y Tecnología (CONICYT) de Chile. Agradezco a los correctores anónimos de la revista por sus útiles y agudos comentarios. 


\section{BiBLIOGRAFÍA}

Aouino, Tomás de (2002). Suma de Teología. Madrid: BAC.

Arancibia, F. (2017). Religión en el Espacio Público. Un estudio desde la filosofía de John Finnis. Santiago, Chile: Ed. Olejnik.

ARIstóteles (1957). On the soul; Parva Naturalia; On breath. Cambridge, Mass.: Harvard University Press.

ARIstóteles (1961). Parts of animals; Movement of animals; Progression of animals. Cambridge, Mass.: Harvard University Press.

Aristóteles (1985). Ética Nicomáquea. Ética Eudemia (Julio Pallí Bonet, trad.). Madrid: Gredos.

Armstrong, D. (1978a). Universals and Scientific Realism. Vol. 1: Nominalism and Realism. Cambridge: Cambridge University Press.

Armstrong, D. (1978b). Universals and Scientific Realism. Vol. 2: A Theory of Universals. Cambridge: Cambridge University Press.

Armstrong, D. (1983). What is a Law of Nature? Cambridge: Cambridge University Press. Armstrong, D. (1989). Universals. An Opinionated Introduction. Boulder: Westview.

Austin, J. (1995). [1832] The province of jurisprudence determined (Wilfrid E. Rumble, ed.). Cambridge: Cambridge University Press.

George, R. P. (1992). «Natural Law and Human Nature».En George, R. P. (Ed.) Natural Law Theory. Contemporary Essays. Oxford: Clarendon Press.

George, R. P. (1999). In Defense of Natural Law. Oxford: Oxford University Press.

Grisez, G. (1965). "The First Principle of Practical Reason. A Commentary on the Summa Theologiae 1-2, Question 94, Article 2». En Natural Law Forum, 10, pp. 168-196.

FinNis, J. (1980). Natural Law and Natural Rights. Oxford: Clarendon Press.

FinNis, J. (1983). Fundamentals of Ethics. Washington D. C.: Georgetown University Press.

FinNis, J. (2000). Ley Natural y Derechos Naturales (Cristóbal Orrego S. trad.). Buenos Aires: Abeledo Perrot.

Gomez-Loвo, A. (2002). Morality and the Human Goods. An Introduction to Natural Law Ethics. Washington D.C.: Georgetown University Press.

Gonzalez, A. M. (2006). Moral, Razón y Naturaleza. Una investigación sobre Tomás de Aquino. Pamplona: EUNSA.

Hart, H. L. A. (1963). The Concept of Law. Oxford: Clarendon Press.

Hume, D. (1896). A Treatise of Human Nature (L. A. Selby-Bigge, ed.). Oxford: Oxford University Press.

Hume, D. (1981). Tratado de la Naturaleza Humana (Felix Duque, trad.). Madrid: Editora Nacional S. A.

Hume, D. (1983). An Enquiry Concerning the Principles of Morals (J. W. Schneewind, ed.). Indiana: Hackett Publishing Company.

Hume, D. (2007). An Enquiry Concerning Human Understanding (Peter Millican, ed.). Oxford: Oxford University Press.

Hume, D. (2014). Investigación sobre los Principios de la Moral (3ra ed.) (Carlos Mellizo, trad.). Madrid: Alianza.

Kelsen, H. (1994). Teoría Pura del Derecho. Introducción a la ciencia del Derecho (Moisés Nilve, trad.). Buenos Aires: EUDEBA.

Lewis, D. (1986). «New Work for a Theory of Universals». En Australasian Journal of Philosophy, Vol. 61, No. 4, pp. 343-377. 
Livingstone, D. W. (1984). Hume's Philosophy of Common Life. Chicago: University of Chicago Press.

Loux, M. J. (2006). Metaphysics. A Contemporary Introduction. London: Routledge.

Mackie, J. L. (1980). Hume's Moral Theory. London: Routledge.

Mackie, J. L. (2000). [1977] Ética. La invención de lo bueno y lo malo (Tomás Fernández, trad.). Barcelona: Gedisa.

Miller, A. (2003). An Introduction to Contemporary Metaethics. Malden: Polity.

Orrego, C. (2000). «Estudio Preliminar». En FINNIS, John, Ley Natural y Derechos Naturales (Cristóbal Orrego S. trad.). Buenos Aires: Abeledo Perrot.

Orrego, C. (2005). Analítica del Derecho Justo. La crisis del positivismo juridico y la crítica del derecho natural. México: UNAM.

RABAdE, S. (1998). «Hume, filosofo de la vida ordinaria: sentir y creer». En ARdanaz, M., Lopez, G., Martin, F. et al. David Hume. Perspectivas sobre su obra. Madrid: Editorial Complutense, pp. 11-24.

Raz, J. (1980). The Concept of a Legal System. Oxford: Clarendon Press.

Rodriguez-Pereyra, G. (2002). Resemblance Nominalism. A solution to the problem of universals. Oxford: Oxford University Press.

STRoud, B. (1977). Hume. London: Routledge.

Sturgeon, N. (2008) «Hume's Metaethics: Is Hume a MoralNoncognitivist?». En RaDCLIfFE, E. (ed.), A Companion to Hume. Oxford: Blackwell.

TAsset, J. L. (1999). «Estudio Introductorio». En Hume, David (1999) Resumen del Tratado de la Naturaleza Humana. Barcelona: Literatura y Ciencia S. L.

Vigo, Alejandro (2007). Aristóteles. Una introducción. Santiago: IES.

Wolfe, Ch. (2006). Natural Law Liberalism. Cambridge: Cambridge University Press.

Universidad Bernardo OHiggins

Fernando Arancibia C.

Facultad de Ciencias Sociales

Departamento de Ciencias del Derecho.

Pontificia Universidad Catolica de Chile

Facultad de Filosofía

Instituto de Filisofía

fnarancibia@uc.cl

[Artículo aprobado para publicación en diciembre de 2015] $]^{96}$

96 Nota editorial: Recordamos que este artículo, como todos los publicados en PENSAMIENTO, fue revisado antes de su edición por el autor, realizando en este caso una pertinente actualización bibliográfica. 